\title{
Lower body temperature in sleeping supine infants
}

\author{
R G North, S A Petersen, M P Wailoo
}

\begin{abstract}
Night time rectal temperature recordings were made from 103 infants sleeping in their own home in different sleeping positions. In most cases sleeping position was verified by video monitoring throughout the night. In the period before an adult-like night time body temperature pattern appeared there was no significant effect of sleeping position upon night time body temperature, in line with previous reports. Once an adult-like night time temperature pattern appeared, infants sleeping supine reached significantly lower rectal temperatures than those sleeping prone or lateral. Babies sleeping supine moved significantly more during the night and were more likely to uncover their hands and arms. These findings suggest that supine sleepers are in a different physiological condition from those sleeping prone or lateral, which may be associated with their lower vulnerability to sudden unexpected infant death. (Arch Dis Child 1995; 72: 340-342)
\end{abstract}

Keywords: infant body temperature, sleeping position.

Sleeping position affects the risk of sudden infant death, with a gradient of risk from prone, which is highest, through lateral to supine positions. ${ }^{12}$ It has been suggested that these differences may be associated in some way with thermoregulation. ${ }^{3}$ In a previous paper we compared the overnight body temperature patterns of babies sleeping prone and not prone. ${ }^{4}$ At that time, too few babies slept supine to permit separate analysis of the lateral and supine positions. Comparing young $(<12$ weeks) and older infants separately we found very little evidence for an effect of sleeping position on body temperature patterns. We now know, however, that body temperature patterns develop in a characteristic way and the timing varies from infant to infant. ${ }^{56}$ For a number of weeks body temperature falls to about $36 \cdot 8^{\circ} \mathrm{C}$ during night sleep, but then, within a few days, it begins to fall to about $36.3^{\circ} \mathrm{C}$, a pattern maintained thereafter unless the baby becomes ill. The age of this transition in normal infants varies from 7 weeks to about 16 weeks. We should therefore consider data separately from babies before and after the 'adult-like' temperature pattern has appeared. In addition, since the 'Back to Sleep' campaign intervened to persuade parents to place babies supine to sleep, a steadily increasing fraction of infants have been sleeping on their backs. During the period since the campaign began we have noticed a gradual fall in the minimum sleeping body temperature of babies monitored as a part of our ongoing studies, and have wondered whether this might be associated with the change in position.

In this paper therefore, we have compared the body temperature pattern of babies sleeping in different positions before and after the adult-like night time body temperature pattern has developed. The sleeping position was mostly verified by continuous video recording throughout the night of monitoring.

\section{Methods}

Subjects were recruited from those born at Leicester maternity hospitals. The data described here were collected over the years 1989-92 as a part of other ongoing work. As far as possible similar numbers of infants sleeping in each position were studied from each year and season to minimise any confounding effects of climatic conditions. As virtually no babies now sleep prone, data from later years of our studies have not been included. The data are completely separate from those used in our previous study of sleeping position and temperature patterns. Parents were approached soon after the birth, and informed consent obtained to monitor their infants at home every week from 6 to about 16 weeks of age. Full perinatal data were obtained for each infant.

At each home visit, the infant was weighed naked and a soft probe inserted $5 \mathrm{~cm}$ from the anal margin to monitor rectal temperature, which was then logged continuously from late afternoon through to the next morning via a Grant Squirrel data logger set to sample once a minute. A second probe connected to another channel on the logger monitored ambient temperature in the baby's bedroom. Parents were also provided with a diary to record, prospectively, all interactions with the baby, such as feeds, nappy changes, etc. In many cases heart rate and oxygen saturation were monitored with a Nellcor NL200 pulse oximeter. Only rectal temperature data are reported here. The parents were asked to record the position in which the baby was put to sleep, and note was also taken of the items of clothing and bedding used, so that a thermal insulation or 'tog' value could be calculated from tables provided by the Shirley Institute, Manchester. Ethical permission was obtained for the monitoring.

Most of the babies were continuously observed throughout at least one night of monitoring using an infrared sensitive camera (Insight Vision Ltd) connected to a long play video recorder. The video recordings allow us 
Details of the infants studied

\begin{tabular}{|c|c|c|c|c|c|}
\hline $\begin{array}{l}\text { Stage of development/ } \\
\text { sleeping position }\end{array}$ & $\begin{array}{l}\text { No of } \\
\text { recordings }\end{array}$ & $\begin{array}{l}\text { Mean (SEM) age } \\
\text { at recording (weeks) }\end{array}$ & $\begin{array}{l}\text { Mean (SEM) minimum } \\
\text { room temperature }\left({ }^{\circ} \mathrm{C}\right)\end{array}$ & $\begin{array}{l}\text { Mean (SEM) tog value } \\
\text { of clothing and wrapping }\end{array}$ & $\begin{array}{l}\text { Year in which } \\
\text { recordings were made }\end{array}$ \\
\hline \multicolumn{6}{|c|}{ Before adult-like pattern } \\
\hline Prone & 12 & $12.42(1.03)$ & $18.75(1.56)$ & $10 \cdot 62(0 \cdot 88)$ & \multirow{3}{*}{$\begin{array}{l}\text { 1990: } 12 \\
\text { 1990: } 10 \\
\text { 1991: } 3 \\
\text { 1990: } 8 \\
\text { 1991: } 4\end{array}$} \\
\hline Lateral & 13 & $10.85(0.53)$ & $19.00(0.67)$ & $11.36(1.46)$ & \\
\hline Supine & 12 & $14.33(0.41)$ & $20 \cdot 16(0.61)$ & $9 \cdot 74(1 \cdot 46)$ & \\
\hline \multicolumn{6}{|c|}{ After adult-like pattern } \\
\hline Prone & 20 & $16 \cdot 25(0 \cdot 26)$ & $17 \cdot 38(1 \cdot 07)$ & $10.63(0.88)$ & \multirow{3}{*}{$\begin{array}{l}\text { 1990: } 18 \\
\text { 1991: } 2 \\
\text { 1990: } 16 \\
\text { 1991: } 5 ; 1992: 2 \\
\text { 1990: } 15 \\
\text { 1991: } 9 ; 1992: 1\end{array}$} \\
\hline Lateral & 21 & $13.89(0.40)$ & $18 \cdot 13(0 \cdot 60)$ & $10.49(0.96)$ & \\
\hline Supine & 25 & $17 \cdot 12(0 \cdot 30)$ & $17.40(0.94)$ & $11 \cdot 68(1.08)$ & \\
\hline
\end{tabular}

to verify sleeping position throughout the night, and to test the accuracy of parental reporting of sleeping position. The video tapes were also scanned and scored in a standard way for movements of the infant.

All data from the loggers were downloaded to computer for analysis, and scanned for evidence of problems such as lost probes, etc. Only unblemished data were analysed further. Temperature curves were compared by two way analysis of variance with repeated measures.

\section{Results}

Data were collected from recordings made on 103 infants, 51 of whom were male. The mean (SEM) birth weight was 2950 (78) $\mathrm{g}$ and gestation $38.07(0.45)$ weeks. The mean (SEM) maternal age was $27 \cdot 7(0.4)$ years and 36 of the infants were first babies. The social class distribution of the families resembled that of the Leicestershire population.

From analysis of the temperature recordings made over all the weeks of monitoring, it is clear that the infants developed body temperature patterns in the way we have previously described $^{56}$ with an infantile pattern of body temperature fall to about $36 \cdot 8^{\circ} \mathrm{C}$ suddenly converting to an adult-like pattern around 12 weeks old. We have therefore collated data separately for infants before and after the adult-like pattern appeared. Within each

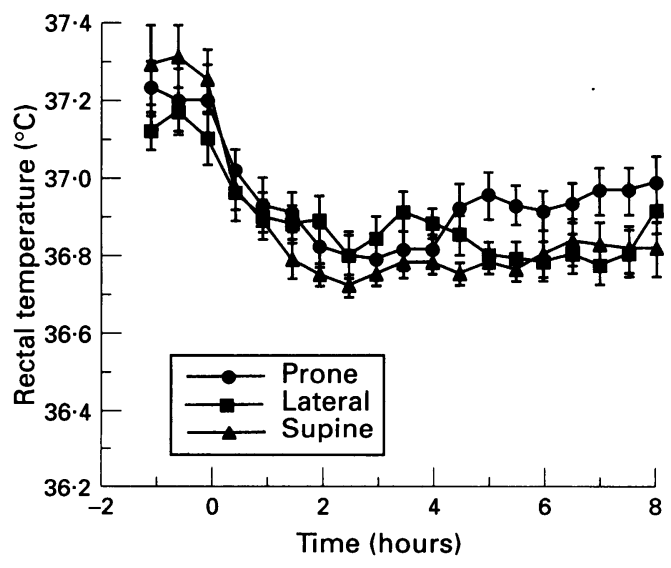

Figure 1 Overnight rectal temperature pattern of babies who have yet to develop and adult-like body temperature pattern, sleeping prone, lateral, or supine. The points show mean (SEM). Bedtime is time zero. Data were collated from 12 babies sleeping prone, 13 sleeping lateral, and 12 sleeping supine. There are no significant differences between the curves (two way analysis of variance). group, the data were separated for babies sleeping prone, lateral (left or right) and supine. 'Prone' was defined as sleeping with the ventral surface of the trunk flat on the mattress, usually with the head on one side. 'Supine' was defined as sleeping with the dorsal surface of the trunk flat on the mattress, usually with the face upwards. 'Lateral' was anything in between. Infants were classified by the sleeping position during the first two hours of the night. The table indicates the number of recordings analysed for each position at each stage of development, together with the average room temperature, tog values, and ages of the babies involved. There were no significant differences in the thermal environments of babies sleeping in different positions.

Parental recording of sleeping position at bedtime proved extremely accurate. In all cases the reported sleeping position corresponded with that observed on the video tape. Furthermore, no babies placed to sleep supine or prone were observed to change sleeping position during the night. Many infants placed to sleep lateral did change their position, with $28 \%$ moving to the prone position and $21 \%$ moving to fully supine. A number of other babies placed lateral moved towards the supine position but could not become fully supine because of the way in which parents had arranged bedding. Older infants were more likely to change position from lateral.

Figure 1 illustrates the body temperature pattern of infants sleeping in different positions before the adult-like night time temperature pattern had developed. There were no significant differences in body temperature pattern between babies sleeping in different positions, though, as we reported earlier, babies sleeping prone tended to warm up faster at the end of the night. ${ }^{4}$ Prone sleepers also tended to cool less rapidly in the two hours after bedtime.

Figure 2 illustrates the body temperature pattern of infants sleeping in different positions once the adult-like temperature pattern has developed. There were only very small differences between the prone and lateral sleepers, but supine babies were significantly cooler $(\mathrm{F}=14.18$ with $1 ; 766 \mathrm{df}, \mathrm{p}<0.001$, comparing prone and supine, $\mathrm{F}=30.39$ with $1 ; 749 \mathrm{df}$, $\mathrm{p}<0.001$ comparing lateral and supine). Even if the babies who started the night lateral but moved to prone were excluded from the lateral group, the difference between prone and lateral remained small. Analysis of the videotapes also shows that there were significantly 


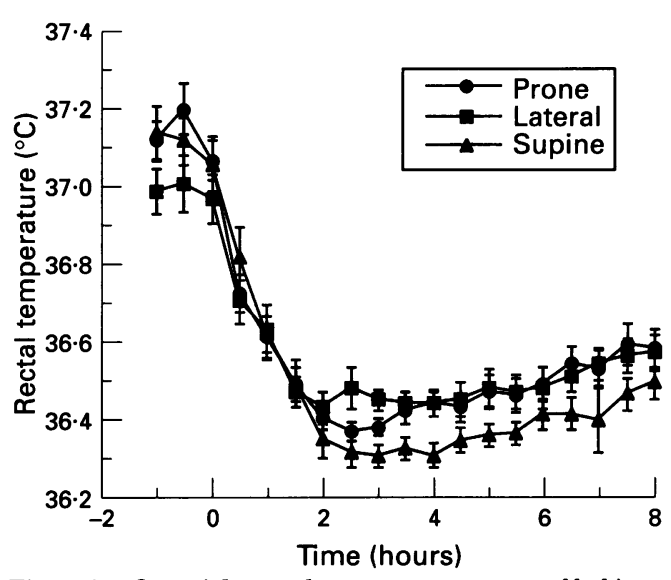

Figure 2 Overnight rectal temperature pattern of babies who have developed an adult-like body temperature pattern, sleeping prone, lateral, and supine. The points show mean (SEM). Data were collated from 20 infants sleeping prone, 21 sleeping lateral, and 25 sleeping supine. The supine infants reached significantly lower rectal temperatures during the night (two way analysis of variance $F=14.18$ with $1,766 \mathrm{df}, p<0.001$ comparing prone and supine, $F=30.39$ with $1,749 d f, p<0.001$ comparing lateral and supine).

more movements detected in infants sleeping supine once the adult-like body temperature pattern had developed.

\section{Discussion}

Once an adult-like night time body temperature had developed babies sleeping on their backs cooled significantly more with sleep at night. This was associated with a greater recorded incidence of movements, particularly of the upper limbs. Neither the ambient temperature nor the thermal insulation of clothing and bedding were significantly different for different sleeping positions, so room temperature or insulation differences cannot account for the different body temperatures.
There are a number of reasons why supine babies might cool more easily. The face will be fully exposed, and movements of the upper limbs will more often lead to the hands being exposed. Indeed we will report elsewhere that prone babies cool significantly more slowly than those sleeping non-prone in the first two hours of the night. It is not, however, clear why supine babies should stabilise at a lower body temperature once the initial cooling has occurred. We must presume that mechanisms of thermoregulation are still in operation, which should lead to physiological changes which mitigate the additional cooling capacity. The fact that this does not occur suggests that the supine infant is in a different physiological condition (perhaps because of altered sleep patterns?), which leads to a different setting of the hypothalamic 'thermostat'. Further investigation into the nature of the altered state may reveal the reason why supine infants are less vulnerable to sudden unexpected death.

The Foundation for the Study of Infant Deaths, Trent Regional Health Authority, and Babes in Arms are thanked for support. The data described here were collected as a part of other, ongoing studies involving a number of research health visitors and they are thanked for their cooperation with the data analysis.

1 Fleming PJ, Gilbert R, Azaz Y, et al. Interaction between bedding and sleeping position in the sudden infant death syndrome: a population based control study. $B M \mathcal{F}$ 1990; 301: 85-9.

2 Mitchell EA, Ford RPK, Taylor BJ, et al. Further evidence supporting a causal relationship between prone sleeping and $9-12$.

3 Nelson EAS, Taylor BJ, Weatherall IL. Sleeping position and infant bedding may predispose to hyperthermia and the sudden infant death syndrome. Lancet 1989; i: 199-200.

4 Petersen SA, Anderson ES, Lodemore M, Rawson D, Wailoo MP. Sleeping position and rectal temperature. Arch Dis Child 1991; 66: 976-80.

5 Lodemore MR, Petersen SA, Wailoo MP. The development of night time temperature rhythms over the first six months of life. Arch Dis Child 1991; 66: 521-4.

6 Lodemore MR, Petersen SA, Wailoo MP. Factors affecting the development of night time temperature rhythms. Arch Dis Child 1992; 67: 1259-61. 